\title{
Fauna invertebrada e atributos físicos e químicos do solo em sistemas de integração lavoura-pecuária
}

\author{
Irzo Isaac Rosa Portilho(1), Rafael Aparecido Crepaldi(2), Clovis Daniel Borges ${ }^{(3)}$, Rogério Ferreira da Silva(4), \\ Júlio César Salton ${ }^{(1)}$ e Fábio Martins Mercante ${ }^{(1)}$
}

\begin{abstract}
(1)Embrapa Agropecuária Oeste, Caixa Postal 661, CEP 79804-970 Dourados, MS. E-mail: irzo_i@terra.com.br, salton@cpao.embrapa.br, mercante@cpao.embrapa.br (2)Universidade Federal da Grande Dourados, Rodovia Dourados-Itahum, Km 12, CEP $79804-970$ Dourados, MS. E-mail: rafaelcrepaldi@ibest.com.br (3)Universidade de São Paulo, Centro de Energia Nuclear na Agricultura, Avenida Centenário, no 303, CEP 13400-970 Piracicaba, SP. E-mail: clovisdb@yahoo.com.br ${ }^{(4)}$ Universidade Estadual de Mato Grosso do Sul, Rua Projetada A, s/nº, CEP 79730-000 Glória de Dourados, MS. E-mail: rogerio@uems.br
\end{abstract}

Resumo - O objetivo deste trabalho foi avaliar o efeito do sistema de integração lavoura-pecuária (ILP) nos principais grupos da macrofauna invertebrada do solo e a relação destes organismos com os atributos físicos e químicos do solo. Foram comparados: sistema de manejo convencional, sistema integração lavoura-pecuária, sistema plantio direto, pastagem cultivada continuamente e fragmentos naturais de Cerrado e de floresta semidecídua. O experimento foi conduzido em Dourados, MS, em um Latossolo Vermelho distroférrico típico, disposto em faixas. A maior densidade, riqueza e diversidade das famílias foi observada no sistema de ILP, em plantio direto, e no sistema em pastagem cultivada continuamente. O sistema convencional promoveu maior redução na comunidade dos organismos invertebrados do solo. O sistema de ILP favorece a manutenção da diversidade da fauna invertebrada, a formação dos agregados estáveis e a fertilidade do solo. A família Sthaphylinidae é um potencial bioindicador de qualidade do solo, principalmente na floresta semidecídua e no sistema de ILP.

Termos para indexação: agroecossistemas, bioindicador, Staphilinidae, rotação de culturas.

\section{Invertebrate fauna and physical and chemical attributes of soil under integrated crop-livestock systems}

\begin{abstract}
The objective of this work was to evaluate the effect of integrated crop-livestock (ICL) systems on the main groups of soil invertebrate macrofauna and the relationship of these organisms with soil physical and chemical attributes. The conventional system, integrated crop-livestock system, no-tillage system, continuously cultivated pasture, and natural fragments of Cerrado and of semi-deciduous forest were compared. The experiment was carried out in Dourados, MS, Brazil, on a Typic Hapludox soil, divided into strips. The highest density, richness, and diversity of families were found in the ICL system, under no-tillage, and in the continuously cultivated pasture. The conventional system caused greater reduction of the soil invertebrate community. The ICL system favors the maintenance of the invertebrate fauna diversity, the formation of stable aggregates, and soil fertility. The Sthaphylinidae family is a potential soil quality bioindicator, especially in the semi-deciduous forest and in the ICL system.
\end{abstract}

Index terms: agroecosystems, bioindicator, Staphilinidae, crop rotation.

\section{Introdução}

O bioma Cerrado, de grande extensão no território brasileiro, vem sendo utilizado intensivamente pelo setor agropecuário para a produção de alimentos (Marchão, 2007). O uso contínuo do solo para produzir grãos e carne bovina tem provocado alterações na composição e na atividade de suas comunidades biológicas (Lavelle, 2002). Portanto, é crescente o interesse pelo biofuncionamento do solo, para melhoria da qualidade ambiental em sistemas de produção agropecuários (Mercante et al., 2008; Silva et al., 2008). Nesse sentido, diversas práticas com manejos conservacionistas foram propostas no cenário agrícola brasileiro, com destaque para o sistema plantio direto e a integração lavoura-pecuária, ambos com rotação de culturas (Aquino et al., 2008).

A utilização de variáveis biológicas, físicas e químicas tem sido indicada para a aferição da qualidade do solo, submetido a diferentes práticas de manejo agrícola e pecuário (Doran \& Parkin, 1994; Salton et al., 2008). Entre os bioindicadores ecológicos, está a

Pesq. agropec. bras., Brasília, v.46, n.10, p.1310-1320, out. 2011 
macrofauna invertebrada do solo, composta por animais com diâmetro corporal acima de $2 \mathrm{~mm}$, tais como minhocas, centopeias, cupins, formigas, aracnídeos, coleópteros em estado larval e adulto, diplópodes, isópodes, entre outros (Lavelle \& Spain, 2001). Esses invertebrados são de fundamental importância para a manutenção da qualidade do solo, pois promovem a redistribuição de nutrientes e de matéria orgânica, por meio de suas atividades biodinâmicas, e participam diretamente do equilíbrio do ecossistema, ao ocupar níveis tróficos da cadeia alimentar (Lee, 1994; Lavelle \& Spain, 2001). A comunidade de organismos da fauna edáfica apresenta uma grande diversidade de formas, comportamentos, tamanhos e estratégias de forrageamento (alimentação e escavação), que podem ser influenciados pelo tipo de manejo empregado (Lavelle, 2002; Aquino et al., 2008).

$\mathrm{O}$ manejo de sistemas agropecuários, com uso de coberturas vegetais, tem mostrado benefícios para a sustentabilidade e a manutenção das propriedades do solo (Lourente et al., 2007; Silva et al., 2008). Contudo, existem poucos estudos sobre os parâmetros ecológicos e a relação da dinâmica das comunidades de invertebrados com os atributos físicos e químicos do solo, em sistemas de integração lavoura-pecuária e outros agroecossistemas.

O objetivo deste trabalho foi avaliar o efeito do sistema de integração lavoura-pecuária nos principais grupos da macrofauna invertebrada do solo e a relação destes organismos com os atributos físicos e químicos do solo.

\section{Material e Métodos}

O experimento foi conduzido no campo experimental da Embrapa Agropecuária Oeste, em Dourados, MS $\left(22^{\circ} 14^{\prime}\right.$ S e $\left.54^{\circ} 49^{\prime} W\right)$, na safra $2009 / 2010$. O clima da região é classificado como Cwa, mesotérmico úmido, com verão quente e inverno seco (Fietz \& Fisch, 2008). Os sistemas de manejo do solo foram implantados em 1995, em um Latossolo Vermelho distroférrico, de textura muito argilosa (Amaral et al., 2000). Nove tratamentos foram dispostos em faixas: dois sistemas de integração lavoura-pecuária, três sistemas de plantio direto, sistema convencional, pastagem cultivada continuamente e fragmentos naturais de Cerrado e de floresta semidecídua (Tabela 1).
Inicialmente, foi cultivado milho (Zea mays L.), sem adubação, em todas as faixas, para esgotamento dos nutrientes do solo, tornando-o mais homogêneo. O sistema de integração lavoura-pecuária (ILP) foi subdividido em duas áreas de 4 ha cada: uma ocupada com pastagem de Urochloa decumbens (Stapf) R.D.Webster (Syn. Brachiaria decumbens Stapf) (ILP-a) e outra com lavoura, rotacionada a cada dois anos (ILP-b). Na área ocupada com lavoura, a soja (Glycine $\max$ (L.) Merril) foi conduzida em sistema plantio direto (SPD) no verão, tendo sido rotacionada com aveia-preta (Avena strigosa Schreb.), no inverno. Durante esse período, a aveia-preta foi utilizada como pastagem temporária. O SPD abrangeu uma área de aproximadamente 8,5 ha, subdividida em três faixas de 2,8 ha cada uma, das quais duas foram ocupadas com soja e uma com milho, no verão, tendo sido rotacionadas com aveia-preta, trigo (Triticum aestivum L.) e nabo-forrageiro (Raphanus raphanistrum L.), no outono/inverno, para conter as sequências: soja/nabo-forrageiro/milho (SPD-a), soja/ trigo/soja (SPD-b) e milho/aveia-preta/soja (SPD-c). As culturas de trigo e aveia-preta foram utilizadas para a produção de grãos, enquanto o nabo-forrageiro foi utilizado para a produção de palha. Neste sistema, foram utilizados herbicidas pós-emergentes e outras tecnologias disponíveis para a região, para aumentar a produtividade de grãos e reduzir as perdas do sistema. O sistema convencional (SC), tradicionalmente utilizado na região, com uso de aração e gradagens, foi estabelecido em área de 2 ha, com cultivo de soja, no verão, e de aveia-preta, no outono/inverno. O sistema de pastagem contínua (PC), para pecuária de corte, consistiu de pastagem permanente com $U$. decumbens, em área de aproximadamente 10 ha. Um sistema adjacente, de aproximadamente 5 ha, com vegetação tipo Cerrado (CE), e um fragmento de reserva natural de floresta semidecídua (FS), ambos mantidos intactos, foram incluídos no experimento, como referencial da condição original do solo. Os sistemas com pastagens por bovinos (ILP e PC) foram conduzidos sob pressão de pastejo ajustada para 7\% (7 kg de massa de matéria seca de forragem para $100 \mathrm{~kg}$ de peso vivo por dia).

As amostragens referentes aos atributos biológicos, físicos e químicos do solo foram realizadas em janeiro de 2010, no período de floração das culturas de soja e milho. Nos sistemas avaliados, foram definidos cinco pontos de amostragem equidistantes 
em $30 \mathrm{~m}$, ao longo de um transecto. Em cada ponto, foi avaliado um monólito de solo de $0,25 \times 0,25 \mathrm{~m}$ de largura e $0,3 \mathrm{~m}$ de profundidade, de acordo com as recomendações de Anderson \& Ingram (1993). A comunidade da fauna invertebrada nas amostras de solo foram triadas manualmente, e os organismos, com mais de $10 \mathrm{~mm}$ de comprimento ou com diâmetro corporal superior a $2 \mathrm{~mm}$, foram extraídos e armazenados em solução de álcool a $70 \%$. Com auxílio de lupa binocular, procedeuse à contagem e à identificação dos organismos (Borror \& Delong, 1969; Triplehorn \& Jonnson, 2010), que foram separados, conforme estádio de desenvolvimento, em adultos ou imaturos (larvas).

Após a triagem dos organismos da fauna edáfica, as amostras da camada superior $(0-10 \mathrm{~cm})$ do monólito de solo foram utilizadas para as análises físicas (amostras deformadas) e químicas, de acordo com método adaptado de Claessen (1997). Para determinação do diâmetro médio ponderado(DMP) de agregados, por via úmida, foi utilizado um conjunto de peneiras com malhas de 9,52,
$4,76,2,0,1,0,0,5,0,25,0,125$ e $0,053 \mathrm{~mm}$, conforme Claessen (1997). Os atributos físicos e químicos do solo estão apresentados na Tabela 2.

A caracterização dos grupos foi realizada com base na densidade (número de indivíduos por metro quadrado), na riqueza (número de famílias) e na diversidade (índice de Shannon-Wiener). O índice de Shannon-Wiener foi calculado como:

$$
\mathrm{H}^{\prime}=-\sum_{\mathrm{i}=1}^{\mathrm{S}} \mathrm{p}_{\mathrm{i}} \ln \mathrm{p}_{\mathrm{i}}
$$

em que: H' é o índice de diversidade; $p$ representa as famílias encontradas; e $\mathrm{S}$ é a riqueza específica. Os dados obtidos (x) para densidade foram transformados em $(\mathrm{x}+0,5)^{0,5}$ e comparados pelo teste de Duncan, a $1 \%$ de probabilidade. Os dados obtidos (x) para riqueza e diversidade não foram transformados, e as médias foram comparadas pelo teste de Duncan, a $1 \%$ de probabilidade. As análises estatísticas foram processadas com uso do programa Assistat (Silva \& Azevedo, 2009).

Tabela 1. Culturas utilizadas em rotação/sucessão nos diferentes sistemas de manejo.

\begin{tabular}{|c|c|c|c|c|c|c|c|c|}
\hline Sistemas & $1995 / 2006$ & $2007 / 2008$ & 2008 & $2008 / 2009$ & 2009 & $2009 / 2010$ & 2010 & $2010 / 2011$ \\
\hline$\overline{I L P-a}$ & Milho & Soja & Aveia-preta & Soja & Aveia-preta & \multicolumn{3}{|c|}{-------------Urochloa decumbens ------------- } \\
\hline ILP-b & Milho & \multicolumn{4}{|c|}{ 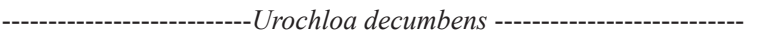 } & \multirow{2}{*}{$\begin{array}{l}\text { Soja } \\
\text { Milho }\end{array}$} & \multirow{2}{*}{$\begin{array}{l}\text { Aveia-preta } \\
\text { Aveia-preta }\end{array}$} & \multirow{2}{*}{$\begin{array}{l}\text { Soja } \\
\text { Soja }\end{array}$} \\
\hline SPD-a & Milho & Soja & Trigo & Soja & Nabo-forrageiro & & & \\
\hline SPD-b & Milho & Milho & Aveia-preta & Soja & Trigo & Soja & Nabo-forrageiro & Milho \\
\hline SPD-c & Milho & Soja & Nabo-forrageiro & Milho & Aveia-preta & Soja & Trigo & Soja \\
\hline $\mathrm{SC}$ & Milho & Soja & Aveia-preta & Soja & Aveia-preta & Soja & Aveia-preta & Soja \\
\hline $\mathrm{PC}$ & Milho & & & --------- $L$ & chloa decumbens & -- & & \\
\hline $\mathrm{CE}$ & ------- & & & Cerrad & 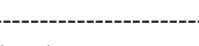 & & & --- \\
\hline FS & & & & loresta se & lecídua & & & \\
\hline
\end{tabular}

ILP-a e ILP-b, sistema de integração lavoura-pecuária; SPD-a, SPD-b e SPD-c, sistema plantio direto; SC, sistema convencional; PC, pastagem contínua; $\mathrm{CE}$, cerrado; e FS, floresta semidecídua.

Tabela 2. Atributos químicos ${ }^{(1)}$ e físicos do solo na camada $0-10 \mathrm{~cm}$ em sistema de integração lavoura-pecuária (ILP-a e ILP-b), plantio direto (SPD-a, SPD-b e SPD-c), convencional (SC), pastagem contínua (PC), Cerrado (CE) e floresta semidecídua (FS).

\begin{tabular}{|c|c|c|c|c|c|c|c|c|c|c|c|c|c|c|c|c|}
\hline Sistema & $\begin{array}{c}\mathrm{pH} \\
\mathrm{em} \mathrm{H}_{2} \mathrm{O}\end{array}$ & $\begin{array}{c}\mathrm{MO} \\
\left(\mathrm{g} \mathrm{dm}^{-3}\right)\end{array}$ & $\begin{array}{c}\mathrm{P} \\
\left(\mathrm{mg} \mathrm{dm}^{-3}\right)\end{array}$ & K & $\begin{array}{l}\mathrm{Ca} \\
--(\mathrm{mm}\end{array}$ & $\begin{array}{l}\mathrm{Mg} \\
\mathrm{l}_{\mathrm{c}} \mathrm{dm}^{-3}\end{array}$ & Al & $\mathrm{Cu}$ & $\mathrm{Fe}$ & $\begin{array}{l}\mathrm{Mn} \\
\mathrm{dm}^{-3}\end{array}$ & $\mathrm{Zn}$ & Areia & $\begin{array}{r}\text { Silte } \\
-(\mathrm{g} \mathrm{kg}\end{array}$ & Argila & Textura & $\begin{array}{l}\text { DMP } \\
(\mathrm{mm})\end{array}$ \\
\hline ILP-a & 5,6 & 42,9 & 18,6 & 0,44 & 5,1 & 2,3 & 0,8 & 12,4 & 35 & 188 & 3,64 & 162 & 148 & 690 & MA & 4,02 \\
\hline ILP-b & 6,4 & 42,5 & 14,3 & 0,38 & 6,2 & 3,2 & 0,1 & 60,5 & 33 & 180 & 1,62 & 179 & 131 & 690 & MA & 3,81 \\
\hline SPD-a & 6,8 & 42,5 & 37,8 & 0,82 & 7,5 & 4,0 & 0,0 & 12,3 & 26 & 194 & 2,2 & 162 & 148 & 690 & MA & 3,27 \\
\hline SPD-b & 6,5 & 37,7 & 69,9 & 0,92 & 7,1 & 3,0 & 0,0 & 13,7 & 26 & 187 & 3,22 & 220 & 127 & 653 & MA & 3,45 \\
\hline SPD-c & 5,9 & 37,2 & 64,2 & 0,80 & 5,3 & 2,3 & 0,7 & 12,2 & 28 & 175 & 2,34 & 179 & 148 & 673 & MA & 2,92 \\
\hline $\mathrm{SC}$ & 6,8 & 27,8 & 21,9 & 0,42 & 8,7 & 2,1 & 0,0 & 18,3 & 29 & 215 & 1,5 & 229 & 131 & 640 & MA & 2,16 \\
\hline $\mathrm{PC}$ & 6,3 & 51,1 & 9,46 & 0,95 & 6,2 & 3,4 & 0,0 & 13,7 & 42 & 174 & 1,6 & 212 & 132 & 656 & MA & 4,91 \\
\hline $\mathrm{CE}$ & 6,5 & 54,2 & 5,02 & 0,87 & 8,9 & 4,1 & 0,0 & 6,44 & 38 & 196 & 1,78 & 221 & 149 & 630 & MA & - \\
\hline FS & 6,7 & 67,7 & 7,34 & 1,94 & 15,0 & 4,5 & 0,0 & 4,36 & 30 & 326 & 2,32 & 163 & 190 & 645 & MA & - \\
\hline
\end{tabular}

${ }^{(1)}$ Determinações adaptadas de Claessen (1997). MA, muito argilosa; DMP, diâmetro médio ponderado dos agregados nos sistemas. 
Utilizou-se o teste de coeficiente de correlação de Pearson para os dados referentes à composição da fauna invertebrada e aos atributos físicos e químicos do solo. As famílias da fauna invertebrada e os atributos físicos e químicos foram submetidos à análise dos componentes principais (ACP). Foram realizadas duas ACP: atributos químicos/parâmetros biológicos e atributos físicos/parâmetros biológicos. Nas duas análises, os ambientais físicos e químicos foram considerados dados de variação suplementar. Os dados foram submetidos à análise de agrupamento (cluster analysis), tendo-se adotado o método do vizinho mais distante (complete linkage), a partir da distância euclidiana, para descrever a similaridade entre os sistemas avaliados. As análises estatísticas foram processadas pelo programa Statistica (Hill \& Lewicki, 2007).

\section{Resultados e Discussão}

Na safra 2009/2010, foram capturados 1.385 indivíduos por metro quadrado, distribuídos em 12 ordens, 21 famílias e três grupos de imaturos (larvas). A maioria das famílias coletadas foi identificada como sendo da ordem Coleoptera (seis), tendo-se considerado adultos e larvas (Tabela 3). A ordem Diptera foi representada por três famílias; as ordens Araneae e Isoptera por duas; e as demais ordens, Hymenoptera, Orthoptera, Dermaptera, Collembola, Thysanura, Geophilida, Blattodea e Tubificina, por uma.

A ordem Coleoptera, abundante na maioria dos solos do Brasil, foi representada pelo maior número de famílias e imaturos (larvas). Embora algumas famílias desta ordem sejam consideradas pragas na agricultura, podem ser benéficas para a fertilidade e a física do solo, principalmente na fase larval (Correia \& Oliveira, 2005). A família Staphylinidae da ordem Coleoptera, presente nos sistemas SPD-c, ILP-a, ILP-b, PC e FS, apresentou maior distribuição relativa no fragmento de floresta (Tabela 4). Cividanes et al. (2009) observaram maior diversidade de Carabidae e Staphylinidae, em área de transição de fragmento florestal, com cultivo, no nordeste do estado de São Paulo. Esses resultados indicam que a família Staphylinidae pode ser um potencial bioindicador da qualidade do solo, pois geralmente está presente em ambientes naturais e mais conservacionistas, com altos teores de matéria orgânica (MO) (Buchs, 2003).

A ordem Diptera e os organismos imaturos (larvas) foram representados por três famílias, presentes nos sistemas SC, SPD-c e FS (Tabela 3). A família Tipulidae da ordem Diptera, representou 5,1\% dos invertebrados do SC (Tabela 4). Os indivíduos desta família são comuns e abundantes em diferentes ambientes e podem causar danos às plantas cultivadas, pois algumas larvas se alimentam de tecido vegetal (Borror \& De Long, 1969). As famílias da ordem Araneae, presentes nos sistemas SPD-a, SPD-c, CE e FS (Tabela 3), são consideradas predadoras e, em geral, alimentam-se de insetos. Esses resultados estão de acordo com os obtidos por Silva et al. (2006), ao usar os mesmos sistemas, na mesma região.

A ordem Isoptera, comum no Cerrado, ocorreu em todos os sistemas avaliados, com exceção do SC (Tabela 3), tendo apresentado grande diversidade e abundância de indivíduos, que podem ser benéficos ao solo, ao atuar como decompositores (Valério et al., 2006). Foi encontrada alta densidade de organismos das famílias Rhinotermitidae e Termitidae, no sistema PC (Tabela 3). Estes organismos têm sido apontados como bioindicadores do estado de degradação do ecossistema, em virtude de sua diversidade taxonômica e ecológica (Brown Junior, 1991). Czepak et al. (2003) observaram a ocorrência desta ordem em sistemas de manejo, como áreas de pastagem cultivada continuamente, mantidos por longos períodos.

Os sistemas em FS e CE não diferiram $(p<0,01)$ dos sistemas ILP, SPD e PC quanto à densidade total de organismos da fauna invertebrada (Tabela 3). O sistema convencional apresentou densidade total significativamente menor que os outros sistemas (Tabela 3), provavelmente em decorrência da menor diversidade de espécies vegetais (sucessão soja/ aveia-preta) e do revolvimento do solo com contínuas arações e gradagens, o que afeta diretamente a estabilidade do ambiente do solo e, consequentemente, a densidade da fauna invertebrada. A redução na quantidade de resíduos culturais, principal fonte de alimento e habitat natural dos organismos, provoca um desequilíbrio entre os invertebrados do solo, o que leva à redução de indivíduos e espécies, até que um novo equilíbrio se estabeleça (Baretta et al., 2006), e afeta a assimilação de nutrientes pelas plantas e a produtividade das culturas. A degradação física e da estrutura biológica do solo, resultante do seu preparo em SC, foi observada na região dos cerrados, em outros estudos (Silva et al., 2006; Salton et al., 2008).

Pesq. agropec. bras., Brasília, v.46, n.10, p.1310-1320, out. 2011 
O SPD-c proporcionou densidade de indivíduos superior à SPD-a, SPD-b, ILP-a e ILP-b. A predominância de organismos das famílias Formicidae (40,5\%) e Enchytraeidae (22,5\%) contribuiu para a alta densidade verificada no SPD-c (Tabela 4). Aquino et al. (2008) também relataram, na mesma área experimental, a presença da família Enchytraeidae, em SPD e ILP. Marchão et al. (2009) observaram alta densidade de organismos considerados engenheiros do solo, como Oligochaetas e Coleopteras, em sistemas de rotação e plantio direto na região do Cerrado. A alta densidade de organismos verificada no sistema em PC está relacionada, principalmente, à presença de indivíduos da ordem Isoptera (Rhinotermitidae e Termitidae), responsável por $66,8 \%$ da densidade total neste sistema (Tabela 4).

Tabela 3. Densidade de indivíduos e riqueza da comunidade de fauna invertebrada, em sistema de integração lavoura-pecuária (ILP-a e ILP-b), plantio direto (SPD-a, SPD-b e SPD-c), convencional (SC), pastagem contínua (PC), Cerrado (CE) e floresta semidecídua (FS).

\begin{tabular}{|c|c|c|c|c|c|c|c|c|c|c|}
\hline \multirow{2}{*}{$\begin{array}{l}\text { Ordem/ } \\
\text { Família }\end{array}$} & \multicolumn{9}{|c|}{ Sistemas avaliados } & \multirow[t]{2}{*}{ Total } \\
\hline & ILP-a & ILP-b & SPD-a & SPD-b & SPD-c & $\mathrm{SC}$ & $\mathrm{PC}$ & $\mathrm{CE}$ & FS & \\
\hline \multicolumn{11}{|l|}{ Coleoptera } \\
\hline Carabidae & 0 & 0 & 1 & 9 & 0 & 4 & 0 & 0 & 0 & 14 \\
\hline Chrysomelidae & 1 & 0 & 0 & 0 & 0 & 0 & 0 & 0 & 0 & 1 \\
\hline Curculionidae & 0 & 2 & 0 & 0 & 0 & 0 & 0 & 0 & 0 & 2 \\
\hline Melolonthidae & 5 & 3 & 0 & 0 & 2 & 0 & 3 & 3 & 5 & 21 \\
\hline Scarabaeidae & 5 & 21 & 0 & 0 & 6 & 0 & 2 & 4 & 3 & 41 \\
\hline Staphylinidae & 5 & 4 & 0 & 0 & 9 & 0 & 2 & 0 & 26 & 46 \\
\hline \multicolumn{11}{|l|}{ Diptera } \\
\hline Chironomidae & 0 & 0 & 0 & 0 & 0 & 0 & 0 & 0 & 2 & 2 \\
\hline Muscidae & 0 & 0 & 0 & 0 & 1 & 0 & 0 & 0 & 0 & 1 \\
\hline Tipulidae & 0 & 0 & 0 & 0 & 0 & 2 & 0 & 0 & 0 & 2 \\
\hline \multicolumn{11}{|l|}{ Araneae } \\
\hline Pholcidae & 0 & 0 & 1 & 0 & 0 & 0 & 0 & 0 & 0 & 1 \\
\hline Zodariidae & 0 & 0 & 0 & 0 & 2 & 0 & 0 & 6 & 6 & 14 \\
\hline \multicolumn{11}{|l|}{ Isoptera } \\
\hline Rhinotermitidae & 39 & 3 & 26 & 3 & 23 & 0 & 113 & 43 & 10 & 260 \\
\hline Termitidae & 0 & 2 & 6 & 2 & 0 & 0 & 12 & 0 & 0 & 22 \\
\hline \multicolumn{11}{|l|}{ Blattodea } \\
\hline Blattidae & 0 & 0 & 0 & 0 & 0 & 0 & 2 & 0 & 6 & 8 \\
\hline \multicolumn{11}{|l|}{ Collembola } \\
\hline Onychiuridae & 0 & 0 & 2 & 0 & 0 & 0 & 0 & 0 & 0 & 2 \\
\hline \multicolumn{11}{|l|}{ Dermaptera } \\
\hline Forficulidae & 8 & 4 & 1 & 0 & 2 & 0 & 3 & 6 & 7 & 31 \\
\hline \multicolumn{11}{|l|}{ Geophilida } \\
\hline Geophilidae & 1 & 0 & 6 & 7 & 6 & 0 & 1 & 9 & 7 & 37 \\
\hline \multicolumn{11}{|l|}{ Hymenoptera } \\
\hline Formicidae & 34 & 20 & 33 & 2 & 128 & 24 & 23 & 25 & 27 & 316 \\
\hline \multicolumn{11}{|l|}{ Orthoptera } \\
\hline Gryllidae & 0 & 0 & 0 & 0 & 0 & 0 & 0 & 2 & 0 & 2 \\
\hline \multicolumn{11}{|l|}{ Thysanura } \\
\hline Lepismatidae & 1 & 0 & 1 & 0 & 0 & 0 & 0 & 2 & 2 & 6 \\
\hline \multicolumn{11}{|l|}{ Tubificina } \\
\hline Enchytraeidae & 43 & 50 & 62 & 43 & 71 & 1 & 22 & 28 & 49 & 369 \\
\hline Anticarsia gemmat & & & & & & & & & & \\
\hline (Hübner, 1818) & 0 & 1 & 0 & 6 & 0 & 2 & 0 & 0 & 0 & 9 \\
\hline Larva/Coleoptera & 4 & 11 & 2 & 3 & 61 & 4 & 4 & 34 & 34 & 157 \\
\hline Larva/Diptera & 3 & 0 & 3 & 1 & 5 & 2 & 0 & 2 & 5 & 21 \\
\hline Densidade $^{(1)}$ & $149 b$ & $121 \mathrm{~b}$ & $144 b$ & $76 b$ & $316 a$ & $39 \mathrm{c}$ & $187 \mathrm{ab}$ & $164 \mathrm{ab}$ & $189 \mathrm{ab}$ & 1.385 \\
\hline Riqueza & 12 & 11 & 12 & 9 & 12 & 7 & 11 & 12 & 14 & 24 \\
\hline
\end{tabular}


Houve maior riqueza da fauna invertebrada na FS, o que não diferiu do CE e do ILP-a, mas foi superior aos demais sistemas de manejo. O SC proporcionou a menor riqueza da fauna invertebrada, não tendo diferido do SPD-b (Tabela 4).

Entre os sistemas manejados, os sistemas de ILP, SPD-a e SPD-c propiciaram maior diversidade de organismos, em relação ao plantio convencional (Tabela 4). Segundo Cordeiro et al. (2004), o tipo de manejo e a modificação da quantidade de resíduos vegetais sobre o solo podem alterar os habitats e a disponibilidade de alimento, o que modifica a diversidade da comunidade da macrofauna edáfica. O sistema de ILP pode ter favorecido as condições de microclima, a disponibilidade de alimento e o habitat no solo para o estabelecimento de novas populações de invertebrados. Em estudo conduzido no Cerrado, Marchão (2007) também observou que o sistema de ILP favorece a diversidade destes organismos no solo. Portanto, as espécies vegetais e os seus processos influenciam diretamente a ocorrência de comunidades de organismos do solo (Aquino et al., 2008). O SC proporcionou a menor diversidade da comunidade da fauna invertebrada do solo, embora não diferindo de SPD-c (Tabela 4). A menor diversidade da fauna em SC pode estar associado à desestruturação do solo e à redução na quantidade de resíduos culturais, que afetam o equilíbrio dos organismos do solo, com redução de inúmeros indivíduos e espécies (Baretta et al., 2006).

$\mathrm{Na}$ ACP, a soma da variabilidade retida nos componentes explicou $56,81 \%$ da variabilidade original dos dados no biplot (Figura 1) referentes aos efeitos dos diferentes manejos sobre a comunidade da fauna

Tabela 4. Distribuição relativa (\%) e parâmetros ecológicos da comunidade de fauna invertebrada em sistema de integração lavoura-pecuária (ILP-a e ILP-b), plantio direto (SPD-a, SPD-b e SPD-c), convencional (SC), pastagem contínua (PC), Cerrado (CE) e floresta semidecídua (FS).

\begin{tabular}{|c|c|c|c|c|c|c|c|c|c|c|}
\hline Família ${ }^{(1)}$ & Hábito $^{(2)}$ & ILP-a & ILP-b & SPD-a & SPD-b & SPD-c & $\mathrm{SC}$ & $\mathrm{PC}$ & $\mathrm{CE}$ & FS \\
\hline Carabidae & Predadores & 0,0 & 0,0 & 0,7 & 11,8 & 0,0 & 10,3 & 0,0 & 0,0 & 0,0 \\
\hline Chrysomelidae & Degradadores & 0,7 & 0,0 & 0,0 & 0,0 & 0,0 & 0,0 & 0,0 & 0,0 & 0,0 \\
\hline Curculionidae & Fitófagos & 0,0 & 1,7 & 0,0 & 0,0 & 0,0 & 0,0 & 0,0 & 0,0 & 0,0 \\
\hline Melolonthidae & Fitófagos / degradadores & 3,4 & 2,5 & 0,0 & 0,0 & 0,6 & 0,0 & 1,6 & 1,8 & 2,6 \\
\hline Scarabaeidae & Degradadores & 3,4 & 17,4 & 0,0 & 0,0 & 1,9 & 0,0 & 1,1 & 2,4 & 1,6 \\
\hline Staphylinidae & Predadores / degradadores & 3,4 & 3,0 & 0,0 & 0,0 & 2,8 & 0,0 & 1,1 & 0,0 & 13,8 \\
\hline Chironomidae & Fitófagos / microfitófagos & 0,0 & 0,0 & 0,0 & 0,0 & 0,0 & 0,0 & 0,0 & 0,0 & 1,0 \\
\hline Muscidae & Degradores & 0,0 & 0,0 & 0,0 & 0,0 & 0,3 & 0,0 & 0,0 & 0,0 & 0,0 \\
\hline Tipulidae & Fitófagos / degradadores & 0,0 & 0,0 & 0,0 & 0,0 & 0,0 & 5,1 & 0,0 & 0,0 & 0,0 \\
\hline Rhinotermitidae & Fitófagos / degradadores & 26,2 & 2,5 & 18,1 & 3,9 & 7,3 & 0,0 & 60,4 & 26,2 & 5,3 \\
\hline Termitidae & Fitófagos / degradadores & 0,0 & 1,7 & 4,2 & 2,6 & 0,0 & 0,0 & 6,4 & 0,0 & 0,0 \\
\hline Pholcidae & Predadores & 0,0 & 0,0 & 0,7 & 0,0 & 0,0 & 0,0 & 0,0 & 0,0 & 0,0 \\
\hline Zodariidae & Predadores & 0,0 & 0,0 & 0,0 & 0,0 & 0,6 & 0,0 & 0,0 & 3,7 & 3,2 \\
\hline Blattidae & Degradadores & 0,0 & 0,0 & 0,0 & 0,0 & 0,0 & 0,0 & 1,1 & 0,0 & 3,2 \\
\hline Enchytraeidae & Degradadores & 28,9 & 41,3 & 43,1 & 56,6 & 22,5 & 2,6 & 11,8 & 17,1 & 25,9 \\
\hline Forficulidae & Degradadores / predadores & 5,4 & 3,3 & 0,7 & 0,0 & 0,6 & 0,0 & 1,6 & 3,7 & 3,7 \\
\hline Formicidae & Fitófagos / predadores & 22,8 & 16,5 & 22,9 & 2,6 & 40,5 & 61,5 & 12,3 & 15,2 & 14,3 \\
\hline Geophilidae & Predadores & 1,0 & 0,0 & 4,2 & 9,2 & 1,9 & 0,0 & 0,5 & 5,5 & 3,7 \\
\hline Gryllidae & Fitófagos & 0,0 & 0,0 & 0,0 & 0,0 & 0,0 & 0,0 & 0,0 & 1,2 & 0,0 \\
\hline Lepismatidae & Degradadores & 0,7 & 0,0 & 0,7 & 0,0 & 0,0 & 0,0 & 0,0 & 1,0 & 1,0 \\
\hline Onychiuridae & Degradadores / microfitófagos & 0,0 & 0,0 & 1,4 & 0,0 & 0,0 & 0,0 & 0,0 & 0,0 & 0,0 \\
\hline $\begin{array}{l}\text { Anticarsia gemmatalis } \\
\text { (Hübner, 1818) }\end{array}$ & Predadores & 0,0 & 0,8 & 0,0 & 7,9 & 0,0 & 5,1 & 0,0 & 0,0 & 0,0 \\
\hline Larva/Coleoptera & Fitófagos / degradadores & 2,7 & 9,1 & 1,4 & 3,9 & 19,3 & 10,3 & 2,1 & 20,7 & 18,0 \\
\hline Larva/Diptera & Fitófagos / degradadores & 2,0 & 0,0 & 2,1 & 1,3 & 1,6 & 5,1 & 0,0 & 1,2 & 2,6 \\
\hline \multicolumn{11}{|l|}{ Parâmetros ecológicos } \\
\hline \multirow{2}{*}{\multicolumn{2}{|c|}{$\begin{array}{l}\text { Riqueza } \\
\text { Índice de Shannon-Wiener }\end{array}$}} & $6,4 \mathrm{ab}$ & $5,6 \mathrm{~b}$ & $6,0 \mathrm{~b}$ & $5,0 \mathrm{bc}$ & $6,2 b$ & $3,2 \mathrm{c}$ & $5,6 b$ & $7,2 \mathrm{ab}$ & $8,4 \mathrm{a}$ \\
\hline & & $2,0 \mathrm{c}$ & $2,0 \mathrm{c}$ & $1,8 \mathrm{de}$ & $1,7 \mathrm{ef}$ & $1,9 \mathrm{~cd}$ & $1,4 \mathrm{~g}$ & $1,6 \mathrm{f}$ & $2,2 b$ & $2,4 \mathrm{a}$ \\
\hline
\end{tabular}

(1)Segundo Borror \& Delong (1969), Righi (1997), Triplehorn \& Jonnson (2010). (2)Hábitos da fauna invertebrada conforme Lavelle \& Kohlmann (1984) e Eisenbeis \& Wichard (1987). Médias seguidas por letras iguais não diferem entre si pelo teste de Duncan, a 1\% de probabilidade. Valores médios de cinco repetições. 
invertebrada e as variáveis químicas do solo, em que CP1 e CP2 retêm, 35,54 e 21,27\%, respectivamente, das informações originais dos dados. No biplot da Figura 2, os diferentes usos do solo influenciaram a comunidade da fauna invertebrada e as variáveis físicas do solo. A soma da variabilidade retida nos componentes explicou $52,89 \%$ da variabilidade original, em que CP1 e CP2 retêm, 33,89 e 18,93\%, respectivamente, das informações originais dos dados.

Os diferentes usos do solo influenciaram a comunidade de invertebrados e os atributos químicos do solo, conforme indicado pelos diferentes grupos formados (Figura 1). O SC e as faixas com manejo no SPD foram agrupados, tendo afetado as famílias Geophilidae, Diptera, Carabidae, Tipulidae, Formicidae e Anticarsia gemmatalis (Hübiner, 1818), além das variáveis químicas $\mathrm{pH}$ e $\mathrm{P}$. O segundo grupo foi formado pela PC e os sistemas de ILP, que influenciaram seis famílias, Scarabaeidae, Chrysomelidae, Curculionidae, Rhinotermitidae, Melolonthidae e Forficulidae e as variáveis químicas $\mathrm{Al}, \mathrm{Fe}$ e $\mathrm{Cu}$. O terceiro grupo foi formado pelos sistemas em CE e FS, agrupados com a maior parte das famílias de invertebrados e com os atributos químicos $\mathrm{Mn}, \mathrm{Mg}, \mathrm{Ca}, \mathrm{K}$ e $\mathrm{MO}$, o que resultou em maior índice de Shannon-Wiener (H') e

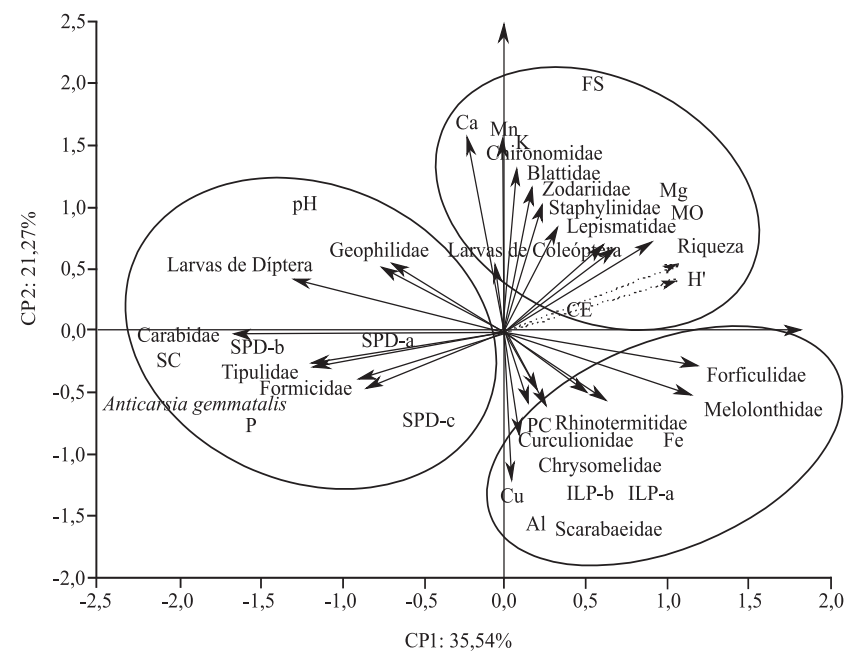

Figura 1. Biplot dos atributos químicos e ecológicos e da composição da comunidade invertebrada do solo em diferentes sistemas de manejo. CP1 e CP2, componentes principais 1 e 2, respectivamente. ILP-a e ILP-b, sistema de integração lavoura-pecuária; SPD-a, SPD-b e SPD-c, sistema plantio direto; SC, sistema convencional; PC, pastagem contínua; CE, Cerrado; FS, floresta semidecídua. riqueza de famílias. Esses resultados indicam que o maior equilíbrio e a diversidade de fatores (habitat, elementos químicos e disponibilidade de alimentação) nos ecossistemas naturais favorece a manutenção da fauna invertebrada do solo.

As famílias da fauna invertebrada edáfica podem ter sido influenciadas pelos fatores químicos do solo, como foi observado nos três grupos (Figura 1). É provável que haja uma ampla interação entre a biologia e a química do solo, com destaque para a família Staphylinidae, que mostrou correlação significativa $(p<0,05)$ com os teores de $\mathrm{MO}, \mathrm{K}, \mathrm{Ca}$ e $\mathrm{Mn}$ e apresentou-se como potencial indicador de qualidade do solo (Tabela 5).

No biplot, os sistemas PC, ILP-a, ILP-b e SPD-a favoreceram a formação e o incremento no DMP, que estão relacionados à textura do solo, especialmente à fração argila (Figura 2). Resultados similares foram obtidos por Salton et al. (2008), em sistemas de pastagem permanente e manejo com rotação de culturas, em MS. Outros fatores, como a presença de raízes e a liberação de exsudatos e polissacarídeos também afetam a agregação do solo (Bronick \& Lal, 2005). A ação biodinâmica da fauna do solo deve ser considerada neste processo, conforme demonstrado por Silva et al. (2006), em estudos conduzidos no mesmo

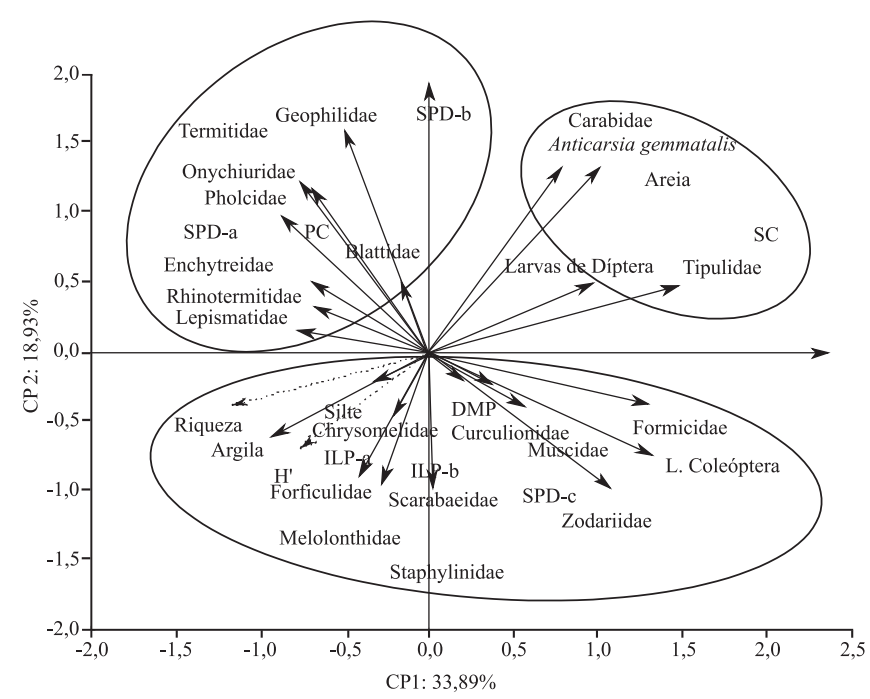

Figura 2. Biplot dos atributos físicos e ecológicos e da composição da comunidade invertebrada do solo em diferentes sistemas de manejo. CP1 e CP2, componentes principais 1 e 2, respectivamente. ILP-a e ILP-b, sistema de integração lavoura-pecuária; SPD-a, SPD-b e SPD-c, sistema plantio direto; SC, sistema convencional; PC, pastagem contínua; DMP, diâmetro médio ponderado. 
local. Os autores verificaram maior densidade de Oligochaetas, classe com várias espécies e famílias na Região Neotropical, de importante papel na estrutura e na aeração do solo, pela formação de galerias biogênicas (Lavelle et al., 1997; Righi, 1997). No Brasil, a maior parte das espécies nativas de Oligochaetas pode estar associada a habitats particulares, como áreas com práticas de manejo orgânico no cultivo de café, que favorecem a diversidade dessas comunidades (Brown \& James, 2007; Bartz et al., 2009). A influência do DMP dos agregados do solo sobre a comunidade da fauna invertebrada nos sistemas de manejo avaliados favoreceu a atividade de determinadas famílias presentes no solo, como Enchytraeidae da ordem Tubificina, e Rhinotermitidae e Termitidae, da ordem Isoptera. Segundo Pringle et al. (2010), a ordem Isoptera contribui para a produtividade do solo, ao estimular a vida vegetal e animal, por meio de redes de colônias uniformemente distribuídas. No presente trabalho, a adoção do SC diminuiu o DMP, em comparação aos demais sistemas (Figura 2). Neste sistema, a maior perturbação dos agregados e a menor quantidade de resíduos adicionados na superfície do solo promoveu incrementos na quantidade de larvas de Diptera, o que também foi observado por Silva et al. (2006).

As famílias Staphylinidae, Chironomidae, Zodariidae e Blattidae apresentaram correlação significativa com MO, K, Ca, Mn, silte e argila (Tabela 5). A família Chrysomelidae apresentou correlação significativa com o teor de Al no solo e pode atuar como um indicador sensível de ambientes com solos com saturação por alumínio. As famílias Rhinotermitidae e Termitidae não apresentaram correlação significativa com Ca. Vendrame et al. (2009) verificaram, em Latossolo do Cerrado, sob pastagem, maior densidade de Isoptera nos solos com baixo teor de $\mathrm{Ca}+\mathrm{Mg}$ trocáveis e maior densidade de Hemiptera e larvas de Coleoptera em solos com maiores teores de carbono total. A família Enchytraeidae da classe Oligochaeta não apresentou correlação significativa com os parâmetros físicos e químicos do solo. Nowak (2001), ao avaliar comunidades de Enchytraeidae em solos mesotróficos e arenosos, observou correlação significativa da diversidade de espécies com o $\mathrm{pH}$ do solo. A família Carabidae apresentou correlação com as áreas com maiores teores de areia. Sistemas de manejo podem proporcionar alterações nos parâmetros biológicos, químicos e físicos do solo, o que pode indicar distúrbios ou equilíbrio no ambiente solo (Mercante et al., 2008; Salton et al., 2008), favorecendo distúrbios ou equilíbrio no ambiente do solo.

Três grupos distintos, G1 (FS e CE), G2 (SPD-a, SPD-c, ILP-a, ILP-b e PC) e G3 (SC e SPD-b), foram formados com base nos dados de riqueza $\mathrm{e}$ diversidade da fauna de invertebrados do solo, a partir da distância euclidiana, tendo apresentado semelhanças entre si (Figura 3). Os sistemas presentes no grupo G1, G2 e G3 apresentaram 78, 58 e 72\% de semelhança entre si. Esses grupamentos podem ser atribuídos às características dos manejos avaliados,

Tabela 5. Coeficientes de correlação de Pearson (r) entre os atributos físicos e químicos e as famílias da fauna invertebrada do solo.

\begin{tabular}{lcccccccccc}
\hline Correlação & Car. & Chr. & Cur. & Scar. & Staph. & Chir. & Rhin. & Zod. & Blat. & Ter. \\
\hline pH & 0,36 & $-0,70$ & $-0,01$ & $-0,17$ & 0,04 & 0,31 & $-0,25$ & 0,21 & 0,29 & 0,38 \\
MO & $-0,58$ & $-0,06$ & $-0,08$ & 0,01 & $0,71^{*}$ & $0,74^{*}$ & 0,32 & $0,74^{*}$ & $0,80^{*}$ & 0,72 \\
P & 0,47 & $-0,14$ & $-0,20$ & $-0,28$ & $-0,31$ & $-0,31$ & $-0,39$ & $-0,43$ & $-0,40$ & $-0,21$ \\
K & $-0,18$ & $-0,32$ & $-0,36$ & $-0,36$ & $0,75^{*}$ & $0,88^{*}$ & 0,02 & 0,64 & $0,89^{*}$ & 0,67 \\
$\mathrm{Ca}$ & 0,01 & $-0,34$ & $-0,20$ & $-0,22$ & $0,72 *$ & $0,90^{*}$ & $-0,27$ & $0,71^{*}$ & $0,82^{*}$ & 0,12 \\
$\mathrm{Mg}$ & $-0,40$ & $-0,40$ & 0,00 & $-0,01$ & 0,39 & 0,54 & 0,17 & 0,67 & 0,56 & $0,81^{*}$ \\
$\mathrm{Al}$ & $-0,32$ & $0,71^{*}$ & $-0,09$ & 0,06 & 0,06 & $-0,20$ & $-0,01$ & $-0,23$ & $-0,27$ & $-0,61$ \\
$\mathrm{Cu}$ & $-0,05$ & $-0,11$ & $0,97 *$ & $0,92^{*}$ & $-0,13$ & $-0,28$ & $-0,27$ & $-0,41$ & $-0,31$ & $-0,10$ \\
Fe & $-0,48$ & 0,19 & 0,09 & 0,20 & $-0,09$ & $-0,16$ & $0,81^{*}$ & 0,19 & 0,08 & 0,43 \\
Mn & $-0,05$ & $-0,12$ & $-0,19$ & $-0,18$ & $0,86^{*}$ & $0,96^{*}$ & $-0,32$ & 0,60 & $0,87 *$ & $-0,36$ \\
Zn & 0,13 & $0,70^{*}$ & $-0,31$ & $-0,23$ & 0,13 & 0,04 & $-0,11$ & $-0,16$ & $-0,07$ & $-0,30$ \\
Areia & $0,80 *$ & $-0,25$ & $-0,14$ & $-0,23$ & $-0,60$ & $-0,46$ & $-0,06$ & $-0,24$ & $-0,43$ & 0,12 \\
Silte & $-0,37$ & $-0,06$ & $-0,15$ & $-0,09$ & $0,94 *$ & $0,97 *$ & $-0,16$ & 0,64 & $0,91^{*}$ & $-0,18$ \\
Argila & $-0,35$ & 0,32 & 0,32 & 0,35 & $-0,57$ & $-0,74$ & 0,26 & $-0,56$ & $-0,70$ & $-0,09$ \\
DMP & $-0,53$ & 0,26 & 0,15 & 0,23 & 0,32 & 0,0 & $0,81^{*}$ & $-0,30$ & 0,70 & 0,65 \\
\hline
\end{tabular}

*Significativo a 5\% de probabilidade. Car., Carabidae; Chr., Chrysomelidae; Curc., Curculionidae; Scar., Scarabaeidae; Staph., Staphylinidae; Chir., Chironomidae; Rhin., Rhinotermitidae; Zod., Zodariidae; Blat., Blattidae; Ter., Termitidae; DMP, diâmetro médio ponderado. 


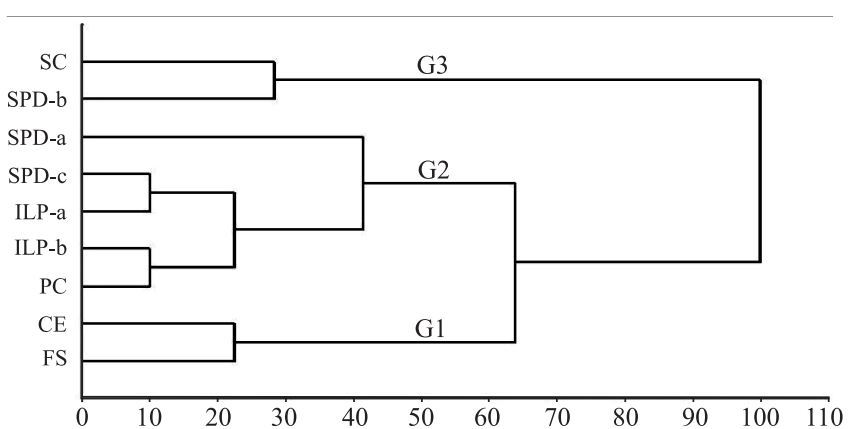

Figura 3. Dendrograma das distâncias entre as comunidades da fauna invertebrada, tendo-se considerado riqueza (número de grupos) e índice de diversidade de Shannon-Wiener, nos sistemas avaliados: sistema de integração lavoura-pecuária (ILP-a e ILP-b), sistema plantio direto (SPD-a, SPD-b e SPD-c), sistema convencional (SC), pastagem contínua (PC), Cerrado (CE) e floresta semidecídua (FS). Grupos formados: G1, G2 e G3.

que proporcionam maior proteção do solo e maior quantidade de resíduos culturais nos manejos com rotação de culturas. De acordo com Schinitzer (1991), os compostos orgânicos, como açúcares, aminoácidos, ceras, fenóis, ligninas e ácidos, que são fonte de alimento de populações de invertebrados, têm origem na decomposição de resíduos vegetais, o que promove um habitat favorável ao estabelecimento desses organismos.

Os grupos G1 e G2 englobam a ligação com distância inferior a $64 \%$, o que permite inferir que a similaridade entre eles é de 36\% (Figura 3). Isso indica que o manejo com rotação de culturas, como os sistemas de ILP e PD, interfere de forma positiva nos parâmetros ecológicos, na riqueza e na diversidade da comunidade de invertebrados da fauna do solo. Portanto, é possível inferir que sistemas de manejo mais conservacionistas favoreçam o equilíbrio dinâmico da fauna invertebrada do solo e, consequentemente, a conservação dos agroecossistemas.

\section{Conclusões}

1. O sistema de integração lavoura-pecuária favorece a manutenção da diversidade da fauna invertebrada, a formação dos agregados estáveis e a fertilidade do solo.

2. Os atributos físicos e químicos do solo interferem na comunidade dos organismos da fauna do solo.
3. A família Sthaphylinidae é um potencial bioindicador de qualidade do solo, com ocorrência principalmente na floresta semidecídua e no sistema de integração lavoura-pecuária.

4. Fragmentos de vegetação nativa de Cerrado e floresta semidecídua favorecem a conservação da biodiversidade da fauna invertebrada do solo.

\section{Agradecimentos}

Ao Conselho Nacional de Desenvolvimento Científico e Tecnológico e ao Projeto Nacional de Ações Integradas Público-Privadas para a Biodiversidade, pelo apoio financeiro.

\section{Referências}

AMARAL, J.A.M. do; MOTCHI, E.P.; OLIVEIRA, H. de; CARVALHO FILHO, A. de; NAIME, U.J.; SANTOS, R.D. dos. Levantamento semidetalhado dos solos do campo experimental de Dourados, da Embrapa Agropecuária Oeste, Município de Dourados, MS. Dourados: Embrapa Agropecuária Oeste; Rio de Janeiro: Embrapa Solos, 2000. 68p. (Embrapa Agropecuária Oeste. Documentos, 22; Embrapa Solos. Documentos, 15).

ANDERSON, J.M.; INGRAM, J.S.I. Tropical soil biology and fertility: a handbook of methods. $2^{\text {nd }}$ ed. Wallingford: CABI, 1993. 240p.

AQUINO, A.M. de; SILVA, R.F. da; MERCANTE, F.M.; CORREIA, M.E.F.; GUIMARÃES, M. de F.; LAVELlE, P. Invertebrate soil macrofauna under different ground cover plants in the no-till system in the Cerrado. European Journal of Soil Biology, v.44, p.91-97, 2008.

BARETTA, D.; SANTOS, J.C.P.; BERTOL, I.; ALVES, M.V.; MANFOI, A.F.; BARETTA, C.R.D.M. Efeito do cultivo do solo sobre a diversidade da fauna edáfica no planalto sul catarinense. Revista de Ciências Agroveterinárias, v.5, p.108-117, 2006.

BARTZ, M.L.C.; BROWN, G.G.; PASINI, A.; FERNANDES, J. de O.; CURMI, P.; DORIOZ, J.; RALISCH, R. Earthworm communities in organic and conventional coffee cultivation. Pesquisa Agropecuária Brasileira, v.44, p.928-939, 2009.

BORROR, D.J.; DELONG, D.M. Introdução ao estudo dos insetos. São Paulo: Edgard Blucher, 1969. 653p.

BRONICK, C.J.; LAL, R. Soil structure and management: a review. Geoderma, v.124, p.3-22, 2005.

BROWN JUNIOR, K.S. Conservation of Neotropical environments: insects as indicators. In: COLLINS, N.M.; THOMAS, J.A. (Ed.). The conservation of insects and their habitats. London: Academic, 1991. p.349-404.

BROWN, G.G.; JAMES, S.W. Biodiversidade, biogeografia e ecologia das minhocas no Brasil. In: BROWN, G.G.; FRAGOSO, C. (Ed.). Minhocas na América Latina: biodiversidade e ecologia. Londrina: Embrapa Soja, 2007. p.297-381. 
BUCHS, W. Biodiversity and agri-environmental indicators-general scopes and skills with special reference to the habitat level. Agriculture, Ecosystems and Environment, v.98, p.35-78, 2003.

CIVIDANES, F.J.; BARBOSA, J.C.; IDE, S.; PERIOTO, N.W.; LARA, R.I.R. Faunistic analysis of Carabidae and Stafhylinidae (Coleoptera) in five agroecosystems in northeastern São Paulo State, Brazil. Pesquisa Agropecuária Brasileira, v.44, p.954-958, 2009.

CLAESSEN, M.E.C. (Org.). Manual de métodos de análise de solo. 2.ed. rev. atual. Rio de Janeiro: Embrapa-CNPS, 1997. 212p. (Embrapa-CNPS. Documentos, 1).

CORDEIRO, F.C.; DIAS, F. de C.; MERLIM, A. de O.; CORREIA, M.E.F.; AQUINO, A.M. de; BROWN, G. Diversidade da macrofauna invertebrada do solo como indicadora da qualidade do solo em sistema de manejo orgânico de produção. Revista Universidade Rural: Série Ciências da Vida, v.24, p.29-34, 2004.

CORREIA, M.E.F.; OLIVEIRA, L.C.M. de. Importância da fauna de solo para a ciclagem de nutrientes. In: AQUINO, A.M. de; ASSIS, R.L. de (Ed.). Processos biológicos no sistema solo-planta: ferramentas para uma agricultura sustentável. Brasília: Embrapa Informação Tecnológica; Seropédica: Embrapa Agrobiologia, 2005. p.77-99.

CZEPAK, C.; ARAÚJO, E.A. de; FERNANDES, P.M. Ocorrência de espécies de cupins de montículo em pastagens no Estado de Goiás. Pesquisa Agropecuária Tropical, v.33, p.35-38, 2003.

DORAN, J.W.; PARKIN, T.B. Defining and assessing soil quality. In: DORAN, J.W.; COLEMAN, D.C.; BEZDICEK, D.F.; STEWART, B.A. (Ed.). Defining soil quality for a sustainable environment. Madison: Soil Science Society of America, 1994. p.3-21. (SSSA. Special publication, 35).

EISENBEIS, G.; WICHARD, W. Atlas on the biology of soil arthropods. Berlin: Springer-Verlag, 1987. 437p.

FIETZ, C.R.; FISCH, G.F. O clima da região de Dourados, MS. 2.ed. Dourados: Embrapa Agropecuária Oeste, 2008. 32p. (Embrapa Agropecuária Oeste. Documentos, 92).

HILL, T.; LEWICKI, P. Statistics methods and aplications. Tulsa: StatSoft, 2007.

LAVELLE, P. Functional domains in soils. Ecological Research, v.17, p.441-450, 2002.

LAVELlE, P.; BIGNELL, D.; LEPAGE, M.; WOLTERS, V.; ROGER, P.; INESON, P.; HEAL, O.W.; DHILlION, S. Soil function in a changing world: the role of invertebrate ecosystem engineers. European Journal of Soil Biology, v.33, p.159-193, 1997.

LAVELlE, P.; KOHLMANN, B. Étude quantitative de la macrofauna du sol dans une forét tropicale humide Du Mexique (Bonampak, Chiapas). Pedobiologia, v.27, p.377-393, 1984.

LAVELlE, P.; SPAIN, A.V. Soil ecology. Dordrecht: Kluwer Academic, 2001. 654p.

LEE, K.E. The biodiversity of soil organisms. Applied Soil Ecology, v.1, p.251-254, 1994.
LOURENTE, E.R.P.; SILVA, R.F. da; SILVA, D.A. da; MARCHETTI, M.E.; MERCANTE, F.M. Macrofauna edáfica e sua interação com atributos químicos e físicos do solo sob diferentes sistemas de manejo. Acta Scientiarum. Agronomy, v.29, p.17-22, 2007.

MARCHÃO, R.L. Integração lavoura-pecuária num latossolo do Cerrado: impacto na física, matéria orgânica e macrofauna. 2007. 134p. Tese (Doutorado) - Universidade Federal de Goiás, Goiânia.

MARCHÃO, R.L.; LAVELLE, P.; CELINI, L.; BALBINO, L.C.; VILELA, L.; BECQUER, T. Soil macrofauna under integrated crop-livestock systems in a Brazilian Cerrado Ferralsol. Pesquisa Agropecuária Brasileira, v.44, p.1011-1020, 2009.

MERCANTE, F.M.; SILVA, R.F. da; FRANCELINO, C.S.F.; CAVALHEIRO, J.C.T.; OTSUBO, A.A. Biomassa microbiana em um Argissolo Vermelho, em diferentes coberturas vegetais, em área cultivada com mandioca. Acta Scientiarum. Agronomy, v.34, p.479-485, 2008.

NOWAK, E. Enchytraeid communities in successional habitats - (from meadow to forest). Pedobiologia, v.45, p.497-508, 2001.

PRINGLE, R.M.; DOAK, D.F.; BRODY, A.K.; JOCQUÉ, R.; PALMER, T.M. Spatial pattern enhances ecosystem functioning in an African Savanna. PLoS Biology, v.8, e1000377, 2010. Available at: <http://www.plosbiology.org/article/ info\%3Adoi\%2F10.1371\%2Fjournal.pbio.1000377>. Accessed on: 2 Jun. 2010.

RIGHI, G. Minhocas da América Latina: diversidade, função e valor. In: CONGRESSO BRASILEIRO DE CIÊNCIA DO SOLO, 26., 1997, Rio de janeiro. Resumos. Rio de Janeiro: Sociedade Brasileira de Ciencia do Solo, 1997. CD ROM.

SALTON, J.C.; MIELNICZUK, J.; BAYER, C.; BOENI, M.; CONCEIÇÃO, P.C.; FABRÍCIO, A.C.; MACEDO, M.C.M.; BROCH, D.L. Agregados e estabilidade de agregados do solo em sistemas agropecuários em Mato Grosso do Sul. Revista Brasileira de Ciência do Solo, v.32, p.11-21, 2008.

SCHINITZER, M. Soil organic matter: the next 75 years. Soil Science, v.151, p.41-58, 1991.

SILVA, F. de A.S.; AZEVEDO, C.A.V. Principal components analysis in the software Assistat-Statistical Attendance. In: WORLD CONGRESS ON COMPUTERS IN AGRICULTURE, 7., 2009, Reno. Proceedings. Reno: American Society of Agricultural and Biological Engineers, 2009. 1 CD ROM.

SILVA, R.F. da; AQUINO, A.M. de; MERCANTE, F.M.; GUIMARÃES, M. de F. Macrofauna invertebrada do solo em sistema integrado de produção agropecuária no Cerrado. Acta Scientiarum. Agronomy, v.30, p.725-731, 2008. Suplemento especial.

SILVA, R.F. da; AQUINO, A.M. de; MERCANTE, F.M.; GUIMARÃES, M. de F. Macrofauna invertebrada do solo sob diferentes sistemas de produção em Latossolo da região do Cerrado. Pesquisa Agropecuária Brasileira, v.41, p.697-704, 2006.

TRIPLEHORN, C.A.; JONNSON, N.F. Estudo dos insetos. São Paulo: Cengage Learning, 2010. 816p. 
VALÉRIO,J.R.;MACEDO,N.;WILCKEN,C.F.;CONSTANTINO, R. Cupins em pastagens, cana-de-açúcar e plantações florestais. In: SALVADORI, J.R.; ÁVILA, C.J.; SILVA, M.T.B. da (Ed.). Pragas de solo no Brasil. Passo Fundo: Embrapa Agropecuária Oeste; Cruz Alta: Fundacep Fecotrigo, 2004. p.409-456.
VENDRAME， P.R.S.; MARCHÃO, R.L.; BRITO, O.R.; GUIMARÃES, M.F.; BECQUER, T. Relationship between macrofauna, mineralogy and exchangeable calcium and magnesium in Cerrado Oxisols under pasture. Pesquisa Agropecuária Brasileira, v.44, p.996-1001, 2009.

Recebido em 30 de junho de 2010 e aprovado em 30 de setembro de 2011 